\title{
NON-EQUILIBRIUM EXCITATION AND IONIZATION \\ OF BE-SEQUENCE IONS
}

\section{By}

\section{J.C. Raymond and A.K. Dupree}

Center for Astrophysics, Harvard College Observatory, and

Smithsonian Astrophysical Observatory, Cambridge, Mass. , U. S.A.

Intensities of resonance and subordinate lines in $\mathrm{C} \mathrm{III} \mathrm{and} \mathrm{O} V$ are calculated for several non-equilibrium states expected to occur in the solar atmosphere. In particular, cooling (recombination) phases of solar flares, and regions of mass flow such as coronal holes and the network offer conditions where non-equilibrium phenomena can be significant. In such situations, the ionization state may not be in equilibrium with the electron temperature. Detailed comparison is made with the ultraviolet observations from the Harvard College Observatory experiment on Skylab which show temporal variations as well as clear non-equilibrium enhancement of the ratio of the $2 s 2 p{ }^{3} \mathrm{P}-2 \mathrm{p}^{2}{ }^{3} \mathrm{P}$ transition to the resonance line $2 \mathrm{~s}^{2}{ }^{1} \mathrm{~s}-2 \mathrm{~s} 2 \mathrm{p}{ }^{1} \mathrm{P}$ in both $\mathrm{C}$ III and $\mathrm{O} \mathrm{V}$. 